\title{
The effect of ovarian cancer screening on sexual activity and functioning: results from the UK collaborative trial of ovarian cancer screening RCT
}

\author{
Lesley Fallowfield ${ }^{\star}, 1$, Ivonne Solis-Trapala ${ }^{2}$, Usha Menon ${ }^{3}$, Carolyn Langridge ${ }^{1}$, Shirley May ${ }^{1}$, Ian Jacobs ${ }^{3,4}$
} and Valerie Jenkins ${ }^{1}$ for and on behalf of the UKCTOCS trialists

${ }^{1}$ Sussex Health Outcomes Research \& Education in Cancer (SHORE-C), Brighton and Sussex Medical School, University of Sussex, Falmer, Brighton BN1 9RX, UK; ${ }^{2}$ Institute for Applied Clinical Sciences, Guy Hilton Research Centre, Keele University, Newcastle ST4 7QB, UK; ${ }^{3}$ Department of Women's Cancer, Institute for Women's Health, University College London, London W1T 7DN, UK and ${ }^{4}$ University of New South Wales, Sydney, NSW 2052, Australia

Background: To examine the impact of multimodal (MMS) and ultrasound (USS) screening on the sexual activity and functioning of 22966 women in the UK Collaborative Trial of Ovarian Cancer Screening (UKCTOCS) RCT.

Methods: Fallowfield's Sexual Activity Questionnaire (FSAQ) was completed prior to randomisation, then annually in a random sample (RS) of women from MMS, USS and control groups. Any women in the study who required repeat screening due to unsatisfactory results formed an Events Sample (ES); they completed questionnaires following an event and annually thereafter.

Results: Over time in the RS $(n=1339)$ there was no difference between the MMS and USS groups in sexual activity compared with controls. In the ES there were significant differences between the USS group $(n=10156)$ and the MMS group $(n=12810)$. The USS group had lower pleasure scores (mean difference $=-0.14, P=0.046$ ). For both groups women who had $\geqslant 2$ repeat screens, showed a decrease in mean pleasure scores compared with their annual scores (mean difference $=-0.16, P=0.005$ ). Similarly mean pleasure scores decreased following more intensive screens compared with annual screening (mean difference $=-0.09, P=0.046$ ).

Conclusions: Ovarian cancer screening did not affect sexual activity and functioning unless a woman had abnormal results and underwent repeated or higher level screening.

Before the introduction of any National screening programme it is important to establish that any of the benefits shown from a wellconducted randomised controlled trial, are weighed against any potential harms of screening. Furthermore individuals invited to join such programmes must be provided with information that allows them to weigh up all these harms and benefits according to their own values and preferences to enable informed decisions about attendance.
The UK Collaborative Trial of Ovarian Cancer Screening (UKCTOCS) is a large randomised trial involving 13 UK centres and over 202000 postmenopausal women. The primary aim is to assess the effect of screening on disease mortality. Volunteers were randomised to annual multimodal screening (MMS) with serum CA125 interpreted using the risk of ovarian cancer algorithm (ROCA), annual transvaginal ultrasound screening (USS), or no screening (control), in a 1:1:2 ratio (Skates et al, 2001; Menon et al,

*Correspondence: Professor Dame L Fallowfield; E-mail: I.j.fallowfield@sussex.ac.uk

Received 19 October 2016; revised 1 February 2017; accepted 22 February 2017; published online 21 March 2017

(C) 2017 Cancer Research UK. All rights reserved 0007-0920/17 
2005). Preliminary results showed a significant mortality reduction with MMS when prevalent cases were excluded, but further follow up is warranted before firm conclusions can be made about the efficacy and cost effectiveness of population screening (Jacobs et al, 2016).

The benefits of screening in terms of early detection and possibility of more effective treatment are well-elucidated, the many putative harms less so. While in other cancer screening programmes, such as those for cervical and breast cancer, falsepositive results led to a high level of anxiety, little is known about the reactions of well women undergoing population screening for ovarian cancer; the sparse literature has focused on those with a familial history (Wardle et al, 1993; Fry et al, 2001). Potential disadvantages of screening for ovarian cancer (OC) include the psychosocial costs of unnecessary surgery and other sequelae resulting from the screening process itself (Bell et al, 1998). The specificity and sensitivity of the process can impact on who presents for screening, their levels of anxiety and adherence, and ultimately the detection and mortality rates for the disease (Robinson et al, 1997). Many factors impact on how women process or filter the information that is presented to them and consequently may impact on levels of anxiety, depression and risk perceptions. Examination therefore of the psychological costs and benefits of the different methods of OC screening in a population screening study of well women is vital.

We have already reported results showing that although OC screening as such did not appear to raise anxiety, psychological morbidity was elevated by more intrusive repeat testing involving transvaginal ultrasound following abnormal annual screens, and also in women diagnosed with OC (Barrett et al, 2014). In addition, withdrawal rates from UKCTOCS for women who required repeat screening were greater in: the USS group compared with the MMS group (20.1 vs 12.9\%), those who required repeat screens early in the study, individuals with a high predisposition to anxiety, and those with high levels of psychological morbidity (Jenkins et al, 2015). We now report the effect of ovarian cancer screening on sexual activity and function.

Sexual dysfunction may result from a complex interplay of psychological, physical and interpersonal relationship factors. Screening may affect the balance of these in numerous ways. It is known for example that women participating in a screening programme for cervical cancer who had abnormal results reported high anxiety levels, mood, concentration and psychosexual disturbances. It is uncertain if these psychosexual problems result from the screening procedures themselves or from the implications of an abnormal result, namely the possibility of a life-threatening disease and necessity for unpleasant treatments (Summers, 1998). Likewise Lerman et al, 1991 reported the impaired psychosexual functioning of women who had an abnormal smear compared with controls. A review by Rogstad, 2002 quotes Quillam's views about the psychological impact of abnormal cytology as 'transforming a well woman with no symptoms into a patient with fears and anxieties'. The review showed that virtually all studies revealed that screening was associated with significant anxiety and psychosexual sequelae as did another by Lewis et al, 2010 who concluded that there is accumulating evidence showing an association between anxiety and depression with sexual dysfunction.

There is less research conducted with women invited for ovarian cancer screening but it seems reasonable to infer from other research that learning that an ovarian cancer screening test was in some way unsatisfactory and needed repeating might raise anxiety which could in turn affect sexual activity. This might be even more apparent in women with a high anxiety trait or predisposition towards anxiety.

In this current report therefore we examined the effect that different methods of OC screening had on sexual activity, specifically:
(1) If there were differences in sexual activity and functioning between the MMS, USS and Control groups that were not explained by anxiety trait levels.

(2) If women who underwent multiple screening experienced a decline in sexual activity compared with that following annual screening.

\section{MATERIALS AND METHODS}

The design of UKCTOCS and the psychosocial study has been described in detail elsewhere (Menon et al, 2008; Barrett et al, 2014). The trial involved 202638 postmenopausal women recruited through 13 UK centres who were randomised to a no screening control group $(n=101359)$ or annual screening using a serum CA125 test in the MMS group $(n=50640)$ or transvaginal ultrasonography (TVS) in the USS group $(n=50639)$. The psychosocial arm of UKCTOCS was approved by the North West MREC committee (ref: MREC 00/8/34).

The blood test for serum CA125 was interpreted using the ROCA; TVS was performed by ultra-sonographers. Repeat screens were categorised as either Level I or II. Level I screens involved repeating annual screens within three months for equivocal results that is, 'intermediate risk' in the MMS group or 'unsatisfactory scan' in the USS group. Level II screens were undertaken within six weeks of abnormal results and involved for the MMS group a repeat CA125 blood test with ROCA and a TVS; those in the USS group had a repeat TVS by a senior ultra-sonographer or consultant. Women were sent letters clarifying their results and reasons for repeat screens.

Events in a population screening trial of essentially healthy women are rare which is why most research is limited to crosssectional studies. As a complete psychosocial assessment of $>200000$ women in UKCTOCS for 6 years was not feasible, we conducted a partial longitudinal follow-up of a cohort of women in the USS and MMS groups who were recalled for at least one repeat screen during their time on study (see Supplementary Consort diagram Figure A). All women had completed questionnaires prior to randomisation at baseline and were only followed up in the psychosocial study after their first recall, and then annually and/or following subsequent repeat screens, provided no abnormality was found. If an abnormality was confirmed, women were referred for a gynaecological oncology opinion with a view to surgery. Those who had both their ovaries removed and/or were diagnosed with ovarian (OC) or other cancer had no further screening. If no ovaries were removed and no cancer detected at surgery, then women could continue in the study.

A further unique feature of the UKCTOCS psychosocial study was identification of a random sample (RS) comprising 1339 women from MMS, USS and control groups monitored for complete longitudinal follow-up. This sample completed psychosocial assessment questionnaires at baseline and following annual screening. In addition to providing complete longitudinal followup, this allowed comparison of psychosocial outcomes between the screened and control groups.

During the psychosocial study period a total 22966 women from the MMS and USS groups in UKCTOCS, including the RS, had at least one repeat screen following annual screening post randomisation. This group of women is hereafter referred to as the Events Sample (ES).

Assessment measures. Study specific questionnaires probing participants' socio-demographic details, attitudes and beliefs about ovarian cancer and their satisfaction with screening and overall psychological morbidity (Fallowfield et al, 2010) were administered. Women also completed the Fallowfield Sexual Activity Questionnaire (FSAQ) (Thirlaway et al, 1996; Atkins 
and Fallowfield, 2007), the Spielberger State/Trait Anxiety Inventory (STAI) (Spielberger et al, 1983) at each time point.

Fallowfield's sexual activity questionnaire (FSAQ). The FSAQ is a validated and widely used questionnaire designed to measure female sexual activity and functioning (Erichsen et al, 2010; Marino et al, 2014; Reif et al, 2015; Da Costa et al, 2016). The FSAQ consists of three sections: Section I enquires whether or not the woman is sexually active; Section II probes reasons for lack of sexual activity by those who are not sexually active; Section III is completed only by sexually active women and measures aspects of sexual function, as well as activity; it has 10 items, which are scored using a Likert format (very much, somewhat, a little, not at all). There are three domains: (i) Pleasure (desire, enjoyment, satisfaction and frequency; (range 0-18). High scores on this subscale indicate greater pleasure resulting from sexual activity; (ii) Discomfort (dryness and pain; (range 0-6). Low scores on this subscale indicate greater discomfort during sexual activity and (iii) Habit (range 0-3) indicates the degree to which the frequency of sexual activity during the previous month was usual for the respondent-'the same', 'not as much', 'somewhat' or 'much more than usual').

Spielberger state/trait anxiety inventory (STAI). The STAI consists of two questionnaires with 20 items rated on simple four point scales. It is a well-known, validated research clinical tool used successfully in many studies to evaluate anxiety proneness (Trait) and the current state of anxiety or anxiety change (State).

Statistical methods. The two primary aims of the analyses were (1) to examine differences in sexual activity and functioning between USS, MMS and control groups over time that were not explained by anxiety trait levels; and (2) to examine the effect of repeat screening and level of this screening on changes in sexual activity. Linear mixed effects and mixed effects logistic regression models were used for the analysis of the FSAQ Section III subscales and individual items. These models extend standard regression analyses to account for the correlation between repeated observations from each individual, through inclusion of a random intercept.

The subscale scores for pleasure and discomfort were analysed with linear mixed effects regression models with a random intercept. Habit was recorded as 1 if frequency of sexual activity was much more or somewhat more than usual and 0 if it was about the same or not as much as usual. Similarly, each individual item of the pleasure score (questions $1,2,4,7,8,10)$ was recorded $(1=$ very much, somewhat, $0=\mathrm{a}$ little, not at all). The individual items of the discomfort score (questions 5,6$)$ were recorded $(1=$ a little, not at all, $0=$ very much, somewhat). The recoded binary variables were analysed using mixed effects logistic regression models with a random intercept.

The regression models examined mean differences between groups and included the following explanatory variables: an indicator of group (MMS, USS or control), baseline age, screening centre and time from baseline represented by calendar year quarters to capture any time periods between assessments shorter than a year. Baseline anxiety trait (low, medium or high) was included to assess whether any differences in sexual functioning may be explained by anxiety trait. An interaction term between group indicator and time was added to examine any differential effects of group membership on the outcome over time.

Three sets of analyses were undertaken for the FSAQ Section III subscales and individual items, based on data collected at baseline and after (i) annual screens for the RS, (ii) annual screens following the first recall for the ES; and (iii) annual screens following the first recall and any subsequent repeats for the ES. The latter analyses included the cumulative number of repeat screens (annual, $1, \geqslant 2$ ) and the level of these (annual, level I or II) as explanatory variables. These were time-varying variables which allowed assessment of the effects of repeat screening and level compared with annual screening on a woman's sexual activity.

Each of the analyses is of clinical interest on its own right and therefore no adjustment was made for multiple hypothesis testing.

Models were fitted using maximum likelihood estimation; $P$ values were calculated using Wald tests. All analyses were performed using the statistical software $\mathrm{R}$ packages 'glmmML' and 'lme4' in R (R Core Team, 2005; Broström, 2013; Bates et al, 2015).

\section{RESULTS}

Findings. The RS comprised 1339 women whose baseline characteristics are described in Table 1 along with those in the ES $(n=22$ 966: USS $=10156$ and MMS $=12810)$. Almost half of the women in each sample, 647 in the RS and 11314 in the ES, indicated that they were sexually active at baseline. A similar distribution was observed by screening group. Table 2 shows that the most common reason given by women who were not sexually active at baseline was 'No partner' with responses ranging from 32 to $39 \%$ across groups and samples. A third of the women in each group and sample indicated that they were not interested in sex. Only $8-13 \%$ women had a physical problem. Table 3 describes baseline frequencies (\%) of those women who were 'somewhat/very much' sexually active in the RS and ES. The distribution of these are similar across groups and samples. Supplementary Tables S1

Table 1. Baseline demographics of Random Sample (RS) and Events Sample (ES)

\begin{tabular}{|c|c|c|c|c|c|}
\hline & \multirow{2}{*}{$\begin{array}{l}\text { Control group } \\
\text { RS }(n=755)\end{array}$} & \multicolumn{2}{|c|}{ Ultrasound (USS) group } & \multicolumn{2}{|c|}{ Multimodal (MMS) group } \\
\hline & & RS $(n=283)$ & $\mathrm{ES}(n=10156)$ & RS $(n=301)$ & $\mathrm{ES}(n=12810)$ \\
\hline Partner: Yes & $597(79.1 \%)$ & 215 (76\%) & $7840(77.2 \%)$ & $234(77.7 \%)$ & 10060 (78.5\%) \\
\hline $\begin{array}{l}\text { Sexually Active } \\
\text { Yes } \\
\text { No }\end{array}$ & $\begin{array}{l}373(49.4 \%) \\
378(50.1 \%)\end{array}$ & $\begin{array}{l}134(47.3 \%) \\
144(50.9 \%)\end{array}$ & $\begin{array}{l}4971(48.9 \%) \\
5124(50.5 \%)\end{array}$ & $\begin{array}{l}140(46.5 \%) \\
159(52.8 \%)\end{array}$ & $\begin{array}{l}6343(49.5 \%) \\
6389(49.9 \%)\end{array}$ \\
\hline Age at randomisation (yrs) mean (sd) & $60.86(6.26)$ & $62.10(6.56)$ & $60.73(6.39)$ & $61.75(6.56)$ & $61.2(6.27)$ \\
\hline $\begin{array}{l}\text { STAl trait anxiety } \\
\text { Low } \\
\text { Medium } \\
\text { High }\end{array}$ & $\begin{array}{l}113(15.1 \%) \\
511(68.4 \%) \\
123(16.5 \%)\end{array}$ & $\begin{array}{r}58(20.8 \%) \\
182(65.2 \%) \\
39(14.0 \%)\end{array}$ & $\begin{array}{l}1668(16.8 \%) \\
6541(66 \%) \\
1697(17.1 \%)\end{array}$ & $\begin{array}{r}58(19.3 \%) \\
187(62.3 \%) \\
55(18.3 \%)\end{array}$ & $\begin{array}{l}2098(16.8 \%) \\
8341(66.7 \%) \\
2065(16.5 \%)\end{array}$ \\
\hline
\end{tabular}


Table 2. Frequency (\%) of reasons for lack of sexual activity at baseline in the random sample and events sample

\begin{tabular}{|c|c|c|c|c|c|c|}
\hline & & Randon & mple $(n=681)$ & & Events samp & $(n=11,513)$ \\
\hline & Reasons for no sex & $\begin{array}{c}\text { Control group } \\
50 \%\end{array}$ & $\begin{array}{c}\text { USS }(n=144) \\
52 \%\end{array}$ & $\begin{array}{c}\mathrm{MM}(n=159) \\
53 \%\end{array}$ & $\begin{array}{c}\text { USS }(n=5124) \\
51 \%\end{array}$ & $\begin{array}{c}\text { MM }(n=6389) \\
50 \%\end{array}$ \\
\hline A & No partner & $138(37 \%)$ & $53(37 \%)$ & $51(32 \%)$ & $1973(39 \%)$ & $2307(36 \%)$ \\
\hline B & Too tired & $34(9 \%)$ & $11(8 \%)$ & $18(11 \%)$ & 459 (9\%) & $572(9 \%)$ \\
\hline C & Partner is tired & $31(8 \%)$ & $13(9 \%)$ & $17(11 \%)$ & $382(7 \%)$ & $487(8 \%)$ \\
\hline D & Not interested in sex & $111(29 \%)$ & $38(26 \%)$ & $48(30 \%)$ & $1527(30 \%)$ & $1933(30 \%)$ \\
\hline $\mathrm{F}$ & I have a physical problem & $48(13 \%)$ & $11(8 \%)$ & $16(10 \%)$ & $573(11 \%)$ & $716(11 \%)$ \\
\hline G & Partner has physical problem & $84(22 \%)$ & $37(26 \%)$ & 47 (30\%) & $1124(22 \%)$ & $1511(24 \%)$ \\
\hline $\mathrm{H}$ & Other reasons & $104(28 \%)$ & $41(28 \%)$ & $53(33 \%)$ & $1470(29 \%)$ & $1831(29 \%)$ \\
\hline
\end{tabular}

Table 3. Baseline frequencies (\%) of 'very much/somewhat' responses to FSAQ Section III questions of random sample (RS) $(n=647)$ and events sample (ES) $(n=11,314)$ who were sexually active

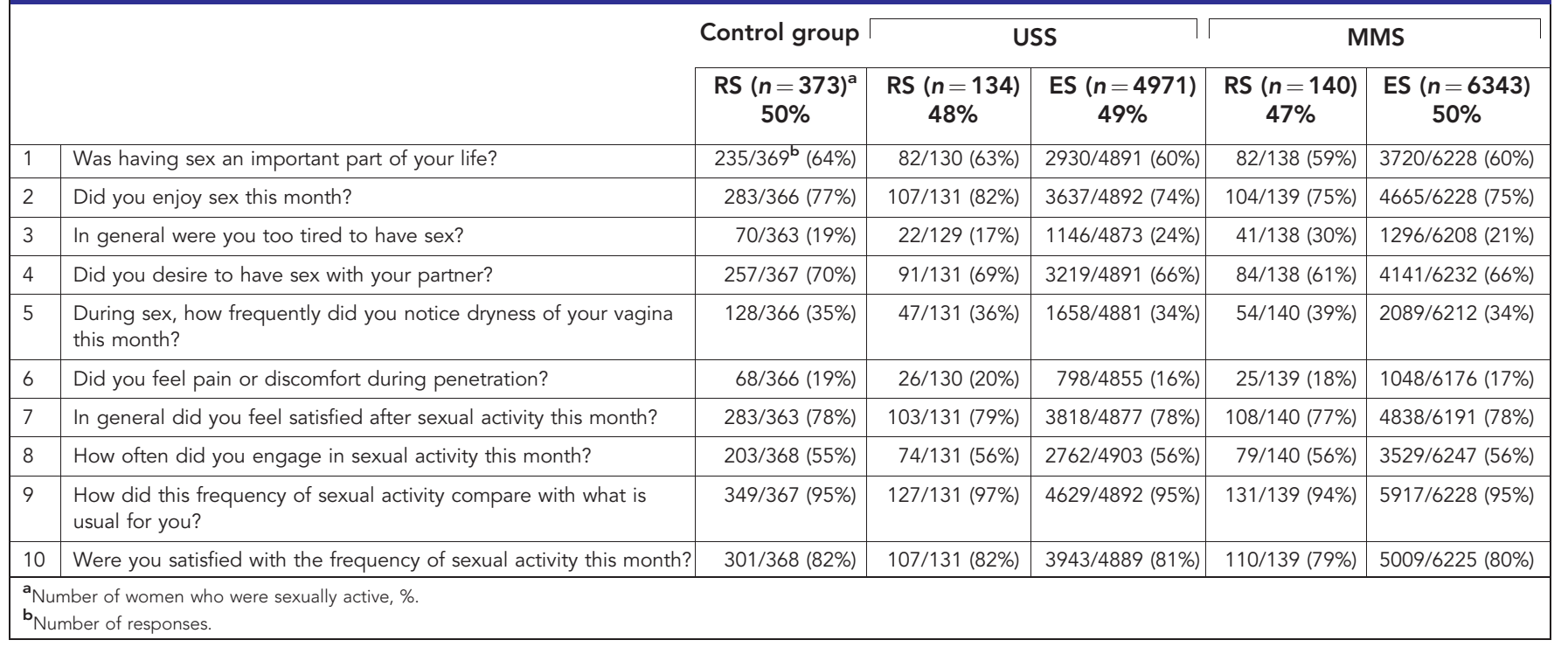

and S2 show the mean and median baseline FSAQ subscales by group. There was a decline in the number of women who were sexually active over time, with the USS and MMS groups showing greater decline than the control group, as shown in Supplementary Tables S3 and S4.

Comparison of sexual function between groups over time. Analysis of annual screen data from the RS who remained sexually active, showed no evidence that sexual function (FSAQ Section III) in the screening groups (MMS or USS) differed from the control group over time. Estimated mean scores differences between MMS and control were $-0.05(P=0.88)$ and $-0.06(P=0.67)$ for pleasure and discomfort respectively, and $\mathrm{OR}=0.99 \quad(P=0.98)$ for habit. When comparing USS with control the estimated mean scores differences were $0.03(P=0.93)$ and $0.09(P=0.60)$ for pleasure and discomfort respectively, and $\mathrm{OR}=1.23(P=0.38)$ for habit. Similarly, analysis of annual screen data from the ES showed no differences between the MMS and USS groups over time. When questionnaires arising from repeat screens were incorporated into the analyses, there were no differences across time between the USS and MMS groups apart from pleasure (mean score difference $=-0.14, P=0.046$ ), which was lower in the USS group over time. There was a moderate interaction between group (MMS or USS) and time (year quarters) on pleasure score

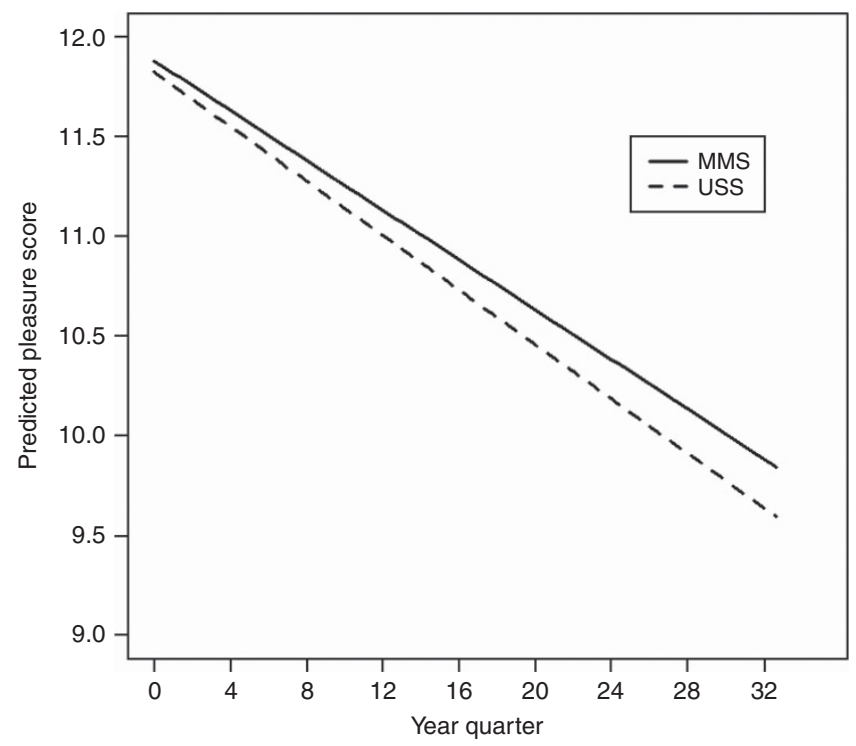

Figure 1. Predicted pleasure score over time by group in the ES using a linear mixed effect model with a random intercept, including group indicator, group indicator by time interaction, age, trait anxiety and centre as explanatory variables. 
$(P=0.09)$. This showed slightly slower decline on the predicted mean score of pleasure in the MMS than in the USS group over time (Figure 1).

Effect of repeat screening and level of screening. There was no difference in mean pleasure score over time in women having 1 repeat screen compared with annual screening $(P=0.73)$. However, women who had $\geqslant 2$ screen repeats had lower mean pleasure scores over time compared with annual screening (mean score difference $=-0.16, P=0.005$ ) (Supplementary Table S5). This was explained mostly from lower satisfaction $(\mathrm{OR}=0.87$, $P=0.07)$, lower enjoyment $(\mathrm{OR}=0.82, P=0.005)$ and lower activity $(\mathrm{OR}=0.77, P<0.001)$. Those who required Level II screens had lower pleasure scores (mean score difference $=-0.09$, $P=0.046)$ which came mostly from lower enjoyment $(\mathrm{OR}=0.89$, $P=0.040)$.

Effect of anxiety, age and time. Women in the ES who had higher levels of trait anxiety at baseline were at higher risk of reduced habit $(\mathrm{OR}=0.57, P<0.001)$ (Figure 2), greater discomfort (mean score difference $=-0.80, P<0.001$ ) and lower pleasure (mean score difference $=-3.20, P<0.001)$. Similar effects were observed in women who had repeat screens and women in the RS having annual screens (Figure 3).

In the ES there was a significant negative effect of age at baseline in pleasure scores. Being one year older was associated with a difference in mean pleasure score of $-0.05(P<0.001)$. For example the mean difference between two women with 10 year age difference at baseline was- $0.50(\times 10-0.05)$. Similarly, having sex was less important $(\mathrm{OR}=0.75, P<0.001)$, there was less enjoyment $(\mathrm{OR}=0.70, P<0.001)$, less desire $(\mathrm{OR}=0.68, P<0.001)$, more dryness $(\mathrm{OR}=0.78, P<0.001)$, less satisfaction $(\mathrm{OR}=0.88$, $P=0.014)$ and lower frequency of sexual activity $(\mathrm{OR}=0.37$, $P<0.001)$. Similar effects were observed when including repeat screens. In the RS older age was associated with less enjoyment $(\mathrm{OR}=0.59, P=0.001)$ and lower frequency $(\mathrm{OR}=0.42, P<0.001)$.

There was deterioration across all FSAQ items over time in both ES and RS. For each year that a woman spent in the study, there was a mean change of pleasure score of $-0.17(P<0.001)$ and-
RS:

trait anxiety level (medium $v$ low): $0.74(0.45,1.22)$ trait anxiety level (high $\vee$ low): $0.70(0.37,1.33)$

ES:

trait anxiety level (medium v low): $0.80(0.70,0.90)$ trait anxiety level (high v low): $0.57(0.49,0.67)$

ES including repeat screens:

trait anxiety level (medium v low): $0.76(0.67,0.86)$ trait anxiety level (high $v$ low): $0.62(0.53,0.73)$

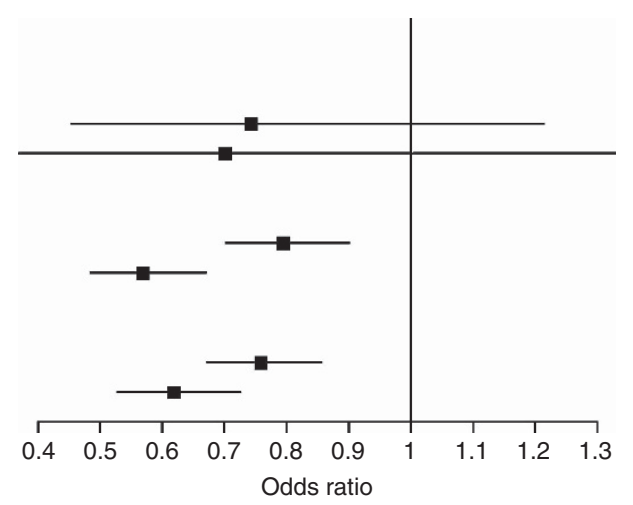

Figure 2. Odds ratios (95\% confidence intervals) of 'somewhat or much more' frequency of sexual activity (Habit) for women with high or medium levels of trait anxiety versus low levels of trait anxiety.

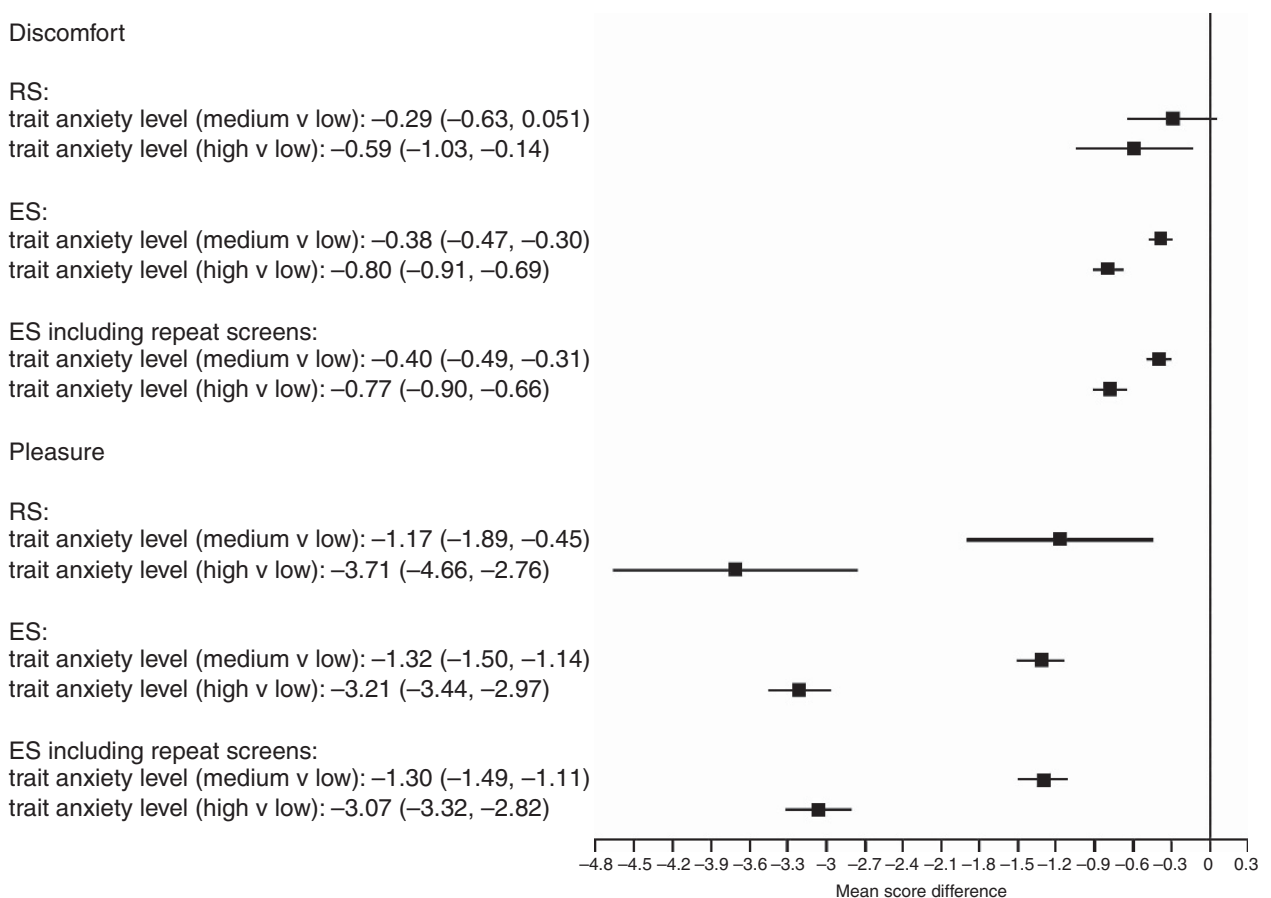

Figure 3. Mean score differences (95\% confidence intervals) of Discomfort and Pleasure among women with high or medium levels of trait anxiety and those with low levels. 
$0.19(P<0.001)$; and a mean change in discomfort score of -0.05 $(P<0.001)$ and $-0.07(P<0.001)$ in the RS and the ES annual screen data, respectively. Similarly, there was a higher risk of reduced habit over time, $\mathrm{OR}=0.82,(P<0.001)$ and $\mathrm{OR}=0.81$ $(P<0.001)$ for the RS and the ES annual screen data, respectively.

\section{DISCUSSION}

Our results show that ovarian cancer screening for postmenopausal women over 50 years of age in itself does not affect sexual activity and functioning. Overall, women experienced a decrease in all aspects of sexual activity across time, as did women with a predisposition to high anxiety. Sexual activity was less frequent, not as pleasurable and more uncomfortable. Cervical screening studies have shown that sexual activity and relationships are affected especially if the woman requires more invasive procedures to determine the results (Juraskova et al, 2007). A similar pattern was recorded in our study. Sexually active women who had two or more repeat screens had lower pleasure scores over time, as did those who required Level II screens involving TVS. Although women in the screening events sample, (MMS and USS) did not differ in sexual activity over time, there were differences in sexual functioning; women in the USS group had significantly lower pleasure scores, based on frequency, enjoyment, desire and satisfaction.

The decision as to whether screening is worthwhile depends on the overall benefits and harms of screening and the resources required. Screening may result in considerable benefits for a small number of people, with larger numbers affected by smaller negative effects. There may also be a range of opinions among women and health professionals regarding the balance of benefits and risks for which they would consider screening valuable.

A recent systematic review reported that a previous 'false-alarm' cancer diagnosis delayed future help seeking behaviours for new or recurrent possible cancer symptoms, in some cases persisting for months or even years (Renzi et al, 2015). We have already reported that the need for repeat screening had an impact on withdrawal from the study and psychological morbidity, especially if it resulted following abnormal rather than equivocal results. Our current report shows that sexual activity is similarly affected. There is therefore a continuing need, irrespective of the OC screening strategy, to explore ways to decrease any unnecessary repeat screens. Also lower use of transvaginal ultrasound with multimodal screening may be less intrusive causing fewer problems. Women with a predisposition to anxiety may also require some extra counselling, help and support if they are to adhere to any screening programme and not experience adverse psychosocial effects from repeat testing.

The results from this extensive psychosocial study together with the UKCTOCS mortality data should be helpful to policy makers deciding whether or not to introduce an ovarian cancer population screening programme.

\section{ACKNOWLEDGEMENTS}

We would like to thank the many volunteers who participated in the trial, the clinicians and research nurses in the centres, data monitors, managers and other academic staff involved over the years. Vernon Farewell has provided statistical support throughout. We appreciate the help of UKCTOCS staff at UCL especially Andy Ryan. This article was funded by Medical Research Council (G9901012 and G0000735) and the Eve Appeal Foundation. The funder had no involvement in the collection, analysis, and interpretation of data; in the writing of the report; and in the decision to submit the paper for publication. LF had full access to the study data and final responsibility for the decision to submit for publication. The main UKCTOCS trial was funded by Medical Research Council, Cancer Research UK, National Institute of Health Research and the Eve Appeal.

\section{CONFLICT OF INTEREST}

IJ and UM have interests in Abcodia Ltd which holds a licence for the ROCA from Queen Mary University of London and Massachusetts General Hospital; their spouses, partners, or children have no financial relationships relevant to the submitted work; and LJF, VJ, CL, SM, IST have no non-financial interests that may be relevant to the submitted work.

\section{AUTHOR CONTRIBUTIONS}

LF, IJ and UM devised the psychosocial study. CL, SM organised the data for analysis, IST analysed the data, VJ, LJF, IST wrote the manuscript. IJ and UM contributed to the manuscript. All authors read and commented on the final version. LJF is the guarantor for the psychosocial data. The study was approved by the North West research ethics committee 20th June 2000 (MREC 00/8/34).

\section{REFERENCES}

Atkins L, Fallowfield LJ (2007) Fallowfield's sexual activity questionnaire in women with without and at risk of cancer. Menopause Int 13(3): 103-109.

Barrett J, Jenkins V, Farewell V, Menon U, Jacobs I, Kilkerr J, Ryan A, Langridge C, Fallowfield L, UKCTOCS trialists (2014) Psychological morbidity associated with ovarian cancer screening: results from more than 23000 women in the randomised trial of ovarian cancer screening (UKCTOCS). BJOG 121(9): 1071-1079.

Bates D, Maechler M, Bolker B, Walker S (2015) Fitting Linear Mixed-Effects Models Using lme4. J Stat Softw 67(1): 1-48.

Bell R, Petticrew M, Sheldon T (1998) The performance of screening tests for ovarian cancer: results of a systematic review. Br J Obstet Gynaecol 105(11): 1136-1147.

Broström G (2013) glmmML: Generalized linear models with clustering. R package version $1.0 \mathrm{https} / /$ CRAN.R-project.org/package $=$ glmmML.

Da Costa FA, Ribeiro MC, Braga S, Carvalho E, Francisco F, Miranda AC, Moreira A, Fallowfield L (2016) Sexual dysfunction in breast cancer survivors: cross cultural adaptation of the sexual activity questionnaire for use in Portugal. Acta Med Port 29(9): 533-541.

Erichsen MM, Husebye ES, Michelsen TM, Dahl AA, Løvås K (2010) Sexuality and fertility in women with Addison's disease. J Clin Endocrinol Metab 95(9): 4354-4360.

Fallowfield L, Fleissig A, Barrett J, Menon U, Jacobs I, Kilkerr J, Farewell V, UKCTOCS Trialists (2010) Awareness of ovarian cancer risk factors, beliefs and attitudes towards screening: baseline survey of 21715 women participating in the UK collaborative Trial of Ovarian Cancer Screening. Br J Cancer 103(4): 454-461.

Fry A, Busby-Earle C, Rush R, Cull A (2001) Prophylactic oophorectomy versus screening: psychosocial outcomes in women at increased risk of ovarian cancer. Psycho-Oncology 10: 231-241.

Jacobs IJ, Menon U, Ryan A, Gentry-Maharaj A, Burnell M, Kalsi JK, Amso NN, Apostolidou S, Benjamin E, Cruickshank D, Crump DN, Davies SK, Dawnay A, Dobbs S, Fletcher G, Ford J, Godfrey K, Gunu R, Habib M, Hallett R, Herod J, Jenkins H, Karpinskyj C, Leeson S, Lewis SJ, Liston WR, Lopes A, Mould T, Murdoch J, Oram D, Rabideau DJ, Reynolds K, Scott I, Seif MW, Sharma A, Singh N, Taylor J, Warburton F, Widschwendter M, Williamson K, Woolas R, Fallowfield L, McGuire AJ, Campbell S, Parmar M, Skates SJ (2016) Ovarian cancer screening and mortality in the UK Collaborative Trial of Ovarian Cancer Screening (UKCTOCS): a randomised controlled trial. Lancet 387(10022): 945-956. 
Jenkins V, Fallowfield L, Langridge C, Barrett J, Ryan A, Jacobs I, Kilkerr J, Menon U, Farewell V (2015) Psychosocial factors associated with withdrawal from the United Kingdom Collaborative Trial Of Ovarian Cancer Screening After 1 episode of repeat screening. Int J Gynecol Cancer 25(8): 1519-1525.

Juraskova I, Butow P, Sharpe L, Campion M (2007) 'What does it mean?' Uncertainty, trust and communication following treatment for precancerous cervical abnormalities. Psychooncology 16: 525-533.

Lerman C, Miller SM, Scarborough R, Hanjani P, Nolte S, Smith D (1991) Adverse psychologic consequences of positive cytologic cervical screening. Am J Obstet Gynecol 165: 658-662.

Lewis RW, Fugl-Meyer KS, Corona G, Hayes RD, Laumann EO, Moreira Jr ED, Rellini AH, Segraves T (2010) Definitions/epidemiology/risk factors for sexual dysfunction. J Sex Med 7(4 Pt 2): 1598-1607.

Marino JL, Saunders CM, Emery LI, Green H, Doherty DA, Hickey M (2014) Nature and severity of menopausal symptoms and their impact on quality of life and sexual function in cancer survivors compared with women without a cancer history. Menopause 21(3): 267-274.

Menon U, Gentry-Maharaj A, Ryan A, Sharma A, Burnell M, Hallett R, Lewis S, Lopez A, Godfrey K, Oram D, Herod J, Williamson K, Seif M, Scott I, Mould T, Woolas R, Murdoch J, Dobbs S, Amso N, Leeson S, Cruickshank D, McGuire A, Campbell S, Fallowfield L, Skates S, Parmar M, Jacobs I (2008) Recruitment to multicentre trials-lessons from UKCTOCS: descriptive study. $\mathrm{Br}$ Med J 337: a2079.

Menon U, Skates SJ, Lewis S, Rosenthal AN, Rufford B, Sibley K, Macdonald N, Dawnay A, Jeyarajah A, Bast Jr RC, Oram D, Jacobs IJ (2005) Prospective study using the risk of ovarian cancer algorithm to screen for ovarian cancer. J Clin Oncol 23(31): 7919-7926.

R Core Team (2005) R: A language and environment for statistical computing. R Foundation for Statistical Computing, Vienna, Austria. URL: https:// www.R-project.org/.
Reif P, Elsayed H, Ulrich D, Bjelic-Radisic V, Häusler M, Greimel E, Tamussino K (2015) Quality of life and sexual activity during treatment of Bartholin's cyst or abscess with a Word catheter. Eur J Obstet Gynecol Reprod Biol 190: 76-80.

Renzi C, Whitaker KL, Wardle J (2015) Over-reassurance and under support after a 'false alarm': a systematic review of the impact on subsequent cancer symptom attribution and help seeking. BMJ Open 5(2): e007002.

Robinson GE, Rosen BP, Bradley LN, Rockert WG, Carr ML, Cole DE, Murphy KJ (1997) Psychological impact of screening for familial ovarian cancer: reactions to initial assessment. Gynecol Oncol 65(2): 197-205.

Rogstad KE (2002) The psychological impact of abnormal cytology and colposcopy. BJOG 109: 364-368.

Skates SJ, Pauler DK, Jacobs IJ (2001) Screening based on the risk of ovarian cancer calculation from Bayesian hierarchical change point and mixture models of longitudinal markers. J Am Stat Assoc 96: 429-439.

Spielberger CD, Gorsuch RL, Lushene R, Vagg PR, Jacobs GA(1983) Manual for the Stait-Trait Anxiety Inventory (Form Y1-Y2). Consulting Psychology Press: Palo Alto, CA, USA.

Summers A (1998) Mental Health consequences of cervical screening. Psychol Health Med 3(1): 113-126.

Thirlaway K, Fallowfield L, Cuzick J (1996) The Sexual Activity Questionnaire: a measure of women's sexual functioning. Qual Life Res 5: 81-90.

Wardle JF, Collins W, Pernet AL, Whitehead MI, Bourne TH, Campbell LS (1993) Psychological impact of screening for familial ovarian cancer. J Natl Cancer Inst 85: 653-657.

This work is published under the standard license to publish agreement. After 12 months the work will become freely available and the license terms will switch to a Creative Commons AttributionNonCommercial-Share Alike 4.0 Unported License.

Supplementary Information accompanies this paper on British Journal of Cancer website (http://www.nature.com/bjc) 